\title{
DEPENDENCE PROPERTIES OF DYNAMIC CREDIT RISK MODELS
}

\author{
NICOLE BÄUERLE* AND UWE SCHMOCK ${ }^{\ddagger}$
}

\begin{abstract}
We give a unified mathematical framework for reduced-form models for portfolio credit risk and identify properties which lead to positive dependence of default times. Dependence in the default hazard rates is modeled by common macroeconomic factors as well as by inter-obligor links. It is shown that popular models produce positive dependence between defaults in terms of association. Implications of these results are discussed, in particular when we turn to pricing of credit derivatives. In mathematical terms our paper contains results about association of a class of non-Markovian processes.
\end{abstract}

\section{INTRODUCTION}

Dependence is an important issue for credit risk models since underestimation of positive dependence may lead to wrong prices for credit derivatives. For example in Szpiro (2009) it is discussed that underestimation of correlation may have contributed to the subprime crisis. In fact, even moderate correlation between defaults may lead to a significant increase in the upper tail of the overall portfolio loss distribution (for an illustration see McNeil et al. (2005) p. 330).

In case the default times of obligors are modeled directly via a copula approach, it may well be possible to discuss the influence of dependence. For example in Burtschell et al. (2008) a comparative analysis of CDO pricing models has been carried out using the concept of stochastic orderings to derive qualitative statements about properties of CDO prices in factor copula models. However, when more complicated stochastic dynamical credit risk models are considered it becomes hard to understand the precise effects of dependence. In Brigo and Capponi (2009) for example the authors discovered a pattern which they called "wrong way risk" where a certain credit risk adjustment decreases in some cases with increasing correlation between the underlying counterparties. Bearing this in mind it is our modest aim to bring some light to the question which features are necessary in stochastic dynamical credit risk models to produce positive dependence. We do not tackle the problem of comparing or quantifying the effect of positive dependence which would then be the next step.

2010 Mathematics Subject Classification. 91G40 (primary); 60E15 (secondary).

Key words and phrases. portfolio credit risk, hazard rate model, reduced-form model, copula, association, concordance order, credit derivatives, credit swaps, stochastic order.

*Financial support from the ESF program AMaMeF is gratefully acknowledged.

$\ddagger$ This work was financially supported by the Christian Doppler Research Association (CDG). The author gratefully acknowledges the fruitful collaboration and support by Bank Austria, the Austrian Federal Financing Agency and COR \& FJA through CDG. 
Indeed from a mathematical point of view it is already challenging to choose the right notion of positive dependence since there are numerous concepts which have been developed for this purpose. Besides association there are among others the notions of block association, positive orthant dependence, positive supermodularity, conditionally increasing, conditionally increasing in sequence, $M T P_{2}$ just to name some of them (see e.g. Müller and Stoyan (2002), section 3.10). In principle notions of positive dependence can be constructed from stochastic orders of positive dependence (see e.g. Colangelo et al. (2005)). In this paper we will mainly restrict to the concept of association.

The next challenge is that we do not have a static model where we can consider random vectors but we have to deal with stochastic processes and have to investigate dependence of them. This is still an active field of research. Liggett (2005) has characterized association of Markov processes via their generators. Association of Itô-diffusion processes has been considered in Herbst and Pitt (1991) for homogeneous processes and in Bäuerle and Manger (2010) for inhomogeneous diffusions. In Bäuerle et al. (2008) dependence properties of Lévy processes have been studied. The recent paper Jakubowski and Karlowska-Pik (2011) investigates stochastic processes with independent increments and introduces the concept of block association to study dependence. The notion of association would be too restrictive in this context. The papers Ebrahimi (2002) and Bäuerle and Manger (2010) investigate the implication of positive dependent Itô-diffusions on hitting times. However, when we consider the default indicator process in credit risk models, it is typically not Markovian nor does it possess independent increments. Hence the available results in the literature cannot be applied here.

In this paper we focus on dynamic credit risk models of reduced-form type. This means, the default hazard rate is modeled explicitly without specifying the precise default mechanism. In general there are three sources for positive dependence: common or correlated risk factors, contagion and learning effects. We give a unified mathematical framework for these models incorporating common macroeconomic factors as well as inter-obligor links. Common macroeconomic factors may be specific factor prices (e.g. interest rates), fundamental indices (e.g. DAX) or production cost (e.g. energy prices). Inter-obligor links are typically given when borrowing and lending contracts are involved. Most common examples are interbank lending agreements. It is often observed that the credit spread of bonds issued by non-defaulted banks increases when another bank defaults. This default interaction is called contagion, concentration risk or correlation risk and can be modeled by a jump of the default hazard rate of non-defaulted obligors. For a discussion and the influence of different dependence constructions see e.g. Giesecke and Weber (2004), Azizpour et al. (2010) and Das et al. (2007). The latter paper shows that common risk factors usually cannot fully explain the size of default correlation. Special cases of our generalized framework are conditionally independent default models, copula models, the model of Jarrow and Yu (2001), the general construction in $\mathrm{Yu}(2007)$ and the model of Frey and Backhaus (2008) among others. As an additional feature we allow for simultaneous defaults of part of the portfolio.

We show in general that these models produce positive dependence among default times under some mild assumptions. In particular, interacting hazard rates mostly produce positive dependence. In mathematical terms we have to prove association of a class of stochastic processes which has not been considered so far. 
Moreover, we highlight the consequences of default correlation in general and when it comes to pricing credit derivatives. The case of independent obligors usually serves as a lower bound. As examples we look at bond prices, $k$-th to default swaps and CDOs. The spirit of our study can be compared with Kijima (1998) where a Markov chain model for credit rating classes is considered and the effect of stochastic monotonicity of the Markov chain is studied. We do not claim that the paper contains relevant results for practical purposes, since the bounds obtained from the independent case are in general weak. However it is a challenging and non-trivial mathematical question to choose the right concept of dependence and to establish positive dependence of these stochastic processes which typically appear in the credit risk framework. Moreover the example of the "wrong way risk" in Brigo and Capponi (2009) shows that correlation effects are not always intuitive.

The paper is organized as follows: In Section 2 we introduce two general reducedform models. In Model 1 the default hazard rate is given explicitly, in Model 2 only the cumulative hazard process is displayed. Section 3 summarizes definitions and facts about association, copulas and other dependence notions which are needed later. In Section 4 we show that under certain assumptions both models imply that the default times of the obligors are positively dependent in terms of association. The implications of these results are discussed and several specific examples are given where they apply. Finally in Section 5 we investigate the consequences for credit derivatives like credit swap contracts, $k$-th-to-default swaps and CDOs.

\section{The Models}

At the beginning we consider rather general reduced-form models for portfolio credit risk. Emphasis is put on the dependence modeling. The general framework includes copula models as well as models with interacting default hazard rates. Models like this are constructed as follows: We consider a portfolio of $d$ obligors. $\lambda_{i}(t)$ is the (positiv $\oint^{1}$ and integrable) default hazard rate of obligor $i \in\{1, \ldots, d\}$ at time $t \geq 0$. Using the cumulative default hazard process

$$
\Lambda_{i}(t):=\int_{0}^{t} \lambda_{i}(s) d s, \quad t \geq 0
$$

the default time of obligor $i$ is defined by

$$
\tau_{i}:=\inf \left\{t \geq 0 \mid \Lambda_{i}(t) \geq E_{i}\right\},
$$

where $E_{i}$ is a standard exponentially distributed random variable. Throughout the paper we suppose that $\Lambda_{i}(t)$ is increasing. It is typically assumed that we have $\lim _{t \rightarrow \infty} \Lambda_{i}(t)=\infty$ almost surely, but we do not need this for our analysis which follows. The default indicator process of obligor $i \in\{1, \ldots, d\}$ is then given by

$$
Y_{i}(t)=1_{\left[E_{i}, \infty\right)}\left(\Lambda_{i}(t)\right), \quad t \geq 0,
$$

i. e., $Y_{i}(t)=1$ if obligor $i$ has defaulted by time $t$ and $Y_{i}(t)=0$ otherwise. The default time $\tau_{i}$ can be recovered from the indicator process by the relation

$$
\tau_{i}=\int_{0}^{\infty}\left(1-Y_{i}(s)\right) d s .
$$

In our paper we consider two special models for the default hazard rate. To this end let $\left(\Omega, \mathcal{F},\left(\mathcal{F}_{t}\right), \mathbb{P}\right)$ be a filtered probability space and suppose that $\left(\Psi_{t}\right)=$

\footnotetext{
${ }^{1}$ We use terms like positive, increasing and decreasing in the weak sense.
} 
$\left(\Psi_{1}(t), \ldots, \Psi_{m}(t)\right)$ is an $m$-dimensional $\left(\mathcal{F}_{t}\right)$-adapted background process which contains relevant economic information, e.g. interest rates, stock prices, economic indices, etc. The information generated by the default indicator process $\left(Y_{t}\right)=$ $\left(Y_{1}(t), \ldots, Y_{d}(t)\right)$ is denoted by $\left(\mathcal{H}_{t}\right)$, i.e.

$$
\mathcal{H}_{t}=\sigma\left(\left\{Y_{u}, u \leq t\right\}\right)
$$

The $\sigma$-algebra which contains both, the information of $\left(\mathcal{F}_{t}\right)$ and $\left(\mathcal{H}_{t}\right)$, is denoted by $\left(\mathcal{G}_{t}\right)$ i.e.

$$
\mathcal{G}_{t}=\mathcal{F}_{t} \vee \mathcal{H}_{t}
$$

As far as the random threshold variables $E=\left(E_{1}, \ldots, E_{d}\right)$ are concerned, we suppose that $E_{i}$ is standard exponentially distributed and independent of $\left(\Psi_{t}\right)$. However, we do not necessarily assume that $E_{1}, \ldots, E_{d}$ are independent, but we assume that the dependence is given by a copula function $C_{E}$.

In what follows we consider two different specifications:

Model 1. Here we suppose that the default hazard rate of obligor $i$ at time $t$ is given by $\lambda_{i}(t):=\lambda_{i}\left(t, \Psi_{t}, Y_{t}\right)$. This means it depends on the background process $\Psi=\left(\Psi_{t}\right)$ and on the number and names of defaulted obligors so far but not on the specific time points of default. In particular, $\lambda_{i}=\left(\lambda_{i}(t)\right)$ is $\left(\mathcal{G}_{t}\right)$-adapted.

Since it is known that the degree of dependence which can be achieved by Model 1 is limited (see e.g. Das et al. (2007)), we consider in Model 2 the cumulative hazard rate process directly. Note in particular results in Mai et al. (2011) which show that the lower tail-dependence among default times is zero when we use in Model 1 the very same affine process for $\lambda_{i}$. Models with cumulative hazard rate process have also been considered in Kou and Peng (2009) and Mai and Scherer (2009).

Model 2. In this approach we incorporate two features which are not present in the first model: We allow for jumps in the cumulative hazard rate process $\Lambda=$ $\left(\Lambda_{1}, \ldots, \Lambda_{d}\right)$, which implies that there may be simultaneous defaults in the portfolio, and we suppose that a default may increase the default hazard rate of the other obligors but this effect fades out (stochastically) after some time, i.e. the cumulative hazard rate process at time $t$ does not only depend on the default indicator process at time $t$ but also on the time since previous defaults have occurred. Thus, suppose we have another family $\Gamma=\left(\Gamma_{i, j}\right)_{i, j \in\{1, \ldots, d\}, i \neq j}$ of stochastic processes, independent of all other processes, where $\Gamma_{i, j}\left(t-\tau_{j}\right)$ describes the increase in the cumulative hazard process $\left(\Lambda_{i}(t)\right)$ of obligor $i$ at time $t \geq 0$ in case a default of obligor $j$ already happened at time $\tau_{j} \leq t$. Here we assume that the environment process $\Psi$ and the infection process $\Gamma$ are stochastic processes, whose components have non-negative and increasing paths. For every obligor $i \in\{1, \ldots, j\}$ we put

$$
\Lambda_{i}(t)=\Psi_{i}(t)+\sum_{j \in\{1, \ldots, d\} \backslash\{i\}} 1_{\left\{\tau_{j} \leq t\right\}} \Gamma_{i, j}\left(t-\tau_{j}\right), \quad t \geq 0,
$$

or in terms of the default indicator process $Y=\left(Y_{1}, \ldots, Y_{d}\right)$ given by $(2.3)$,

$$
\Lambda_{i}(t)=\Psi_{i}(t)+\sum_{j \in\{1, \ldots, d\} \backslash\{i\}} Y_{j}(t) \Gamma_{i, j}\left(\int_{0}^{t} Y_{j}(s) d s\right), \quad t \geq 0 .
$$

This is a generalization of the model of Jarrow and Yu (2001) which we will discuss in Subsection 4.6.2. 


\section{PRELIMINARIES}

In this section we summarize definitions and facts about dependence aspects which will be used later.

3.1. Association. Let us recall the concept of association of random vectors which has been introduced by Esary et al. (1967). The association property reflects positive dependence within a random vector. It is widely used in applications and weaker than other well-known dependence concepts (see e.g. Szekli (1995), Müller and Stoyan (2002), Joe (1997)).

Definition 3.1. An $\mathbb{R}^{d}$-valued random vector $X$ as well as its distribution $\mathcal{L}(X)$ are said to be associated, if

$$
\operatorname{Cov}(f(X), g(X)) \geq 0
$$

for all measurable, (componentwise) increasing functions $f, g: \mathbb{R}^{d} \rightarrow \mathbb{R}$ for which $f(X), g(X)$ and their product are integrable.

Association of a random vector $X$ may be established by taking in (3.1) increasing test functions which are binary or bounded and continuous (see Szekli (1995) Section 3.1). Note that in several dimensions, a componentwise increasing function need not be measurable.

An important case where association arises is the case of monotone mixtures of associated random variables. More precisely, suppose that the probability law of the random vector $X$ depends on a random vector $\Theta$.

Definition 3.2. The random vector $X=\left(X_{1}, \ldots, X_{d}\right)$ is said to be a monotone mixture of $\Theta=\left(\Theta_{1}, \ldots, \Theta_{k}\right)$ if for every measurable, bounded and componentwise increasing $f: \mathbb{R}^{d} \rightarrow \mathbb{R}$ there exists a measurable, componentwise increasing $h: \mathbb{R}^{k} \rightarrow$ $\mathbb{R}$ such that

$$
h(\Theta) \stackrel{\text { a.s. }}{=} \mathbb{E}[f(X) \mid \Theta] .
$$

The following properties of association will be crucial. For a proof of (a)-(d) see Esary et al. (1967) and for (e) the reader is referred to Jogdeo (1978).

Lemma 3.3. (a) If $X=\left(X_{1}, \ldots, X_{d}\right)$ is associated, then $\left(f_{1}(X), \ldots, f_{k}(X)\right)$ is associated for every $k \in \mathbb{N}$ and all measurable increasing (or decreasing) functions $f_{1}, \ldots, f_{k}: \mathbb{R}^{d} \rightarrow \mathbb{R}$.

(b) If $X_{1}, \ldots, X_{d}$ are independent, then $X=\left(X_{1}, \ldots, X_{d}\right)$ is associated.

(c) If $X=\left(X_{1}, \ldots, X_{d}\right)$ and $Y=\left(Y_{1}, \ldots, Y_{k}\right)$ are associated and stochastically independent, then $\left(X_{1}, \ldots, X_{d}, Y_{1}, \ldots, Y_{k}\right)$ is associated.

(d) If $\left\{X_{n}\right\}_{n \in \mathbb{N}}$ is a sequence of associated, $\mathbb{R}^{d}$-valued random vectors converging to $X$ in distribution, then $X$ is again associated.

(e) If the conditional distribution $\mathcal{L}(X \mid \Theta)$ is a.s. associated, $\Theta$ is associated, and $X$ is a monotone mixture of $\Theta$, then the vector $(X, \Theta)$ is associated.

Association can be extended to stochastic processes in a natural way.

Definition 3.4. An $\mathbb{R}^{d}$-valued stochastic process $\left(X_{t}\right)_{t \in I}$ with some non-void index set $I$ is called associated if for all $k \in \mathbb{N}$ and all indices $t_{1}, \ldots, t_{k} \in I$ the $\mathbb{R}^{d k}$-valued random vector $\left(X_{t_{1}}, \ldots, X_{t_{k}}\right)$ is associated. 
Examples for associated processes are given later in Section 4.5. The next lemma shows that the pathwise integration of an associated process yields again an associated process. This will be useful in particular when passing from the default hazard rate to the cumulative default hazard process via 2.1.

Lemma 3.5. Let $(X, Z)$ be an associated process, where $X=\left(X_{t}\right)_{t \geq 0}$ is $\mathbb{R}^{d}$-valued and càdlàg and $Z=\left(Z_{t}\right)_{t \geq 0}$ is $\mathbb{R}^{m}$-valued. Then the process

$$
Y_{t}=\int_{0}^{t} X_{s} d s, \quad t \geq 0
$$

is well defined and the $\mathbb{R}^{2 d+m}$-valued process $(X, Y, Z)$ is associated.

Proof. The idea is to approximate the integral in (3.2) by Riemann sums. Since every path of every component of $X$ is càdlàg, it is also, on every compact interval, bounded and continuous except at a countable number of jump points (only a finite number of these jumps can be larger than a given $\varepsilon>0$ ); hence it is Riemann integrable and $Y$ is well defined.

For every $n \in \mathbb{N}$ define the $\mathbb{R}^{d}$-valued approximating process $\hat{Y}^{n}$ by

$$
\hat{Y}_{t}^{n}=\frac{1}{n} \sum_{l=0}^{\lfloor n t\rfloor-1} X_{l / n}, \quad t \geq 0 .
$$

Consider $k \in \mathbb{N}$ and times $0 \leq t_{1}<\ldots<t_{k}$. Since by assumption the process $(X, Z)$ is associated, we obtain with Definition 3.4 that the vector

$$
\left(\left(X_{t_{1}}, Z_{t_{1}}\right), \ldots,\left(X_{t_{k}}, Z_{t_{k}}\right), X_{0}, X_{1 / n}, \ldots, X_{\left(\left\lfloor n t_{k}\right\rfloor-1\right) / n}\right)
$$

is associated. Hence by Lemma 3.3 (a) the vector

$$
\left(\left(X_{t_{1}}, \hat{Y}_{t_{1}}^{n}, Z_{t_{1}}\right), \ldots,\left(X_{t_{k}}, \hat{Y}_{t_{k}}^{n}, Z_{t_{k}}\right)\right)
$$

is associated. Due to Riemann integrability, the $\mathbb{R}^{(2 d+m) k}$-valued random vectors given in 3.3 converge to the vector $\left(\left(X_{t_{1}}, Y_{t_{1}}, Z_{t_{1}}\right), \ldots,\left(X_{t_{k}}, Y_{t_{k}}, Z_{t_{k}}\right)\right)$ as $n \rightarrow \infty$, which is therefore associated by Lemma 3.3 d). This implies the assertion.

A notion which implies association via Lemma 3.8 a) below and which is sometimes easier to check is conditional increasing in sequence (cf. Müller and Stoyan (2002), section 3.10). It is particularly convenient in case of a Markov process and we will use it in Subsection 4.5.3.

Definition 3.6. A random vector $X=\left(X_{1}, \ldots, X_{d}\right)$ as well as its distribution $\mathcal{L}(X)$ are said to be conditional increasing in sequence (CIS) if for every $k \in$ $\{1, \ldots, d-1\}$ and every bounded increasing $f: \mathbb{R} \rightarrow \mathbb{R}$ there exists a measurable, componentwise increasing $h: \mathbb{R}^{k} \rightarrow \mathbb{R}$ such that

$$
h\left(X_{1}, \ldots, X_{k}\right) \stackrel{\text { a.s. }}{=} \mathbb{E}\left[f\left(X_{k+1}\right) \mid X_{1}, \ldots, X_{k}\right] .
$$

Finally we remark that association implies positive supermodular dependence, which is our main tool for the comparison of credit derivatives prices, see Section 5 . The definition is as follows:

Definition 3.7. $\quad$ (a) A function $f: \mathbb{R}^{d} \rightarrow \mathbb{R}$ is called supermodular if

$$
f(x)+f(y) \leq f(x \vee y)+f(x \wedge y) \quad \text { for all } x, y \in \mathbb{R}^{d},
$$

where $x \vee y$ and $x \wedge y$ denote the componentwise maximum and minimum of $x$ and $y$ respectively. 
(b) Let $X=\left(X_{1}, \ldots, X_{d}\right)$ be an $\mathbb{R}^{d}$-valued random vector and denote by $X^{\perp}=$ $\left(X_{1}^{\perp}, \ldots, X_{d}^{\perp}\right)$ a version with independent components but the same onedimensional marginal distributions, i. e., $X_{i} \stackrel{\mathrm{d}}{=} X_{i}^{\perp}$ for all $i \in\{1, \ldots, d\}$. Then $X$ and its distribution $\mathcal{L}(X)$ are said to be positive supermodular dependent (PSD) if

$$
\mathbb{E}\left[f\left(X^{\perp}\right)\right] \leq \mathbb{E}[f(X)]
$$

for all measurable, supermodular $f: \mathbb{R}^{d} \rightarrow \mathbb{R}$ for which the expectations exist.

We summarize the previously mentioned implications:

Lemma 3.8. Let $X=\left(X_{1}, \ldots, X_{d}\right)$ be an $\mathbb{R}^{d}$-valued random vector.

(a) If $X$ is conditional increasing in sequence (CIS), then $X$ is associated.

(b) If $X$ is associated, then $X$ is positive supermodular dependent.

Proof. For (a) see Müller and Stoyan (2002) Theorem 3.10.11. For part (b) see Christofides and Vaggelatou (2004).

3.2. Copulas. Credit risk models often make use of the copula concept. Therefore it seems to be reasonable to recall the definition of a copula (for an introduction to copulas, see e.g. Nelsen (2006)). Suppose $X=\left(X_{1}, \ldots, X_{d}\right)$ is an $\mathbb{R}^{d}$-valued random vector. Let $F_{1}, \ldots, F_{d}$ denote its marginal, right-continuous distribution functions. We define the copula $C_{X}:[0,1]^{d} \rightarrow[0,1]$ of $X$ to be the distribution function of $\left(F_{1}\left(X_{1}\right), \ldots, F_{d}\left(X_{d}\right)\right)$. Then we have

$$
\begin{aligned}
\mathbb{P}\left(X_{1} \leq x_{1}, \ldots, X_{d} \leq x_{d}\right) & =\mathbb{P}\left(F_{1}\left(X_{1}\right) \leq F_{1}\left(x_{1}\right), \ldots, F_{d}\left(X_{d}\right) \leq F_{d}\left(x_{d}\right)\right) \\
& =C_{X}\left(F_{1}\left(x_{1}\right), \ldots, F_{d}\left(x_{d}\right)\right)
\end{aligned}
$$

for all $\left(x_{1}, \ldots, x_{d}\right) \in \mathbb{R}^{d}$. If $F_{i}$ is continuous, then $F_{i}\left(X_{i}\right)$ is uniformly distributed on the unit interval $[0,1]$. Note that for simplicity reasons we made a special choice for the copula, because in general (3.4) determines $C_{X}$ only on the support of $\left(F_{1}\left(X_{1}\right), \ldots, F_{d}\left(X_{d}\right)\right)$, which is, for example, a finite subset of $[0,1]^{d}$ if $X_{1}, \ldots, X_{d}$ attain only finitely many values. For a (always right-continuous) distribution function $F: \mathbb{R} \rightarrow[0,1]$ define the lower quantile function $F^{\leftarrow}:[0,1] \rightarrow[-\infty, \infty]$ by

$$
F^{\leftarrow}(y)=\inf \{x \in \mathbb{R} \mid F(x) \geq y\}, \quad y \in[0,1],
$$

where inf $\varnothing:=\infty$. It follows from McNeil et al. (2005) Proposition A.4 that

$$
\left(X_{1}, \ldots, X_{d}\right) \stackrel{\text { a.s. }}{=}\left(F_{1}^{\leftarrow}\left(F_{1}\left(X_{1}\right)\right), \ldots, F_{d}^{\leftarrow}\left(F_{d}\left(X_{d}\right)\right)\right)
$$

Since increasing functions of associated random variables are associated by Lemma 3.3 a , we see that association is a property of the copula as defined above. To summarise:

Lemma 3.9. An $\mathbb{R}^{d}$-valued random vector $X$ is associated if and only if its copula $C_{X}$ is associated. 


\section{Association of Default times}

4.1. Implications of associated hazard rates. In this section we will prove that positive dependence between the default hazard rates or the cumulative default hazard processes leads to positive dependence between the default times where positive dependence is expressed in terms of association. In what follows we write $\lambda=(\lambda(t))_{t \geq 0}$ with $\lambda(t)=\left(\lambda_{1}(t), \ldots, \lambda_{d}(t)\right)$ for the $[0, \infty)^{d}$-valued process of joint default hazard rates and $\Lambda=(\Lambda(t))_{t \geq 0}$ with $\Lambda(t)=\left(\Lambda_{1}(t), \ldots, \Lambda_{d}(t)\right)$ for the $[0, \infty)^{d}$-valued process of cumulative joint default hazard rates. Contrary to this name, we will not implicitly assume that $\Lambda$ arises via (2.1), instead we will assume this explicitly when needed. The following lemma is a quite general observation:

Lemma 4.1. (a) If the joint hazard rate process $\lambda$ is associated and has càdlàg paths, then the process $\Lambda$ of cumulative joint hazard rates, given by (2.1), is associated.

(b) If the process $\Lambda$ is associated and has right-continuous paths and if the default thresholds $E=\left(E_{1}, \ldots, E_{d}\right)$ are associated and independent of $\Lambda$, then the default indicator process $Y=\left(Y_{1}, \ldots, Y_{d}\right)$, given in terms of $\Lambda$ and E by (2.3), is associated.

(c) If the default indicator process $Y=\left(Y_{1}, \ldots, Y_{d}\right)$ is associated and every obligor defaults eventually for large $t$, then the vector of default times $\tau=$ $\left(\tau_{1}, \ldots, \tau_{d}\right)$ given in terms of $Y$ by (2.4) is well defined and associated.

Proof. (a) This is a special case of Lemma 3.5 .

(b) For $e=\left(e_{1}, \ldots, e_{d}\right) \in[0, \infty)^{d}$ the map

$$
[0, \infty)^{d} \ni\left(x_{1}, \ldots, x_{d}\right) \mapsto\left(1_{\left[0, e_{1}\right)}\left(x_{1}\right), \ldots, 1_{\left[0, e_{d}\right)}\left(x_{d}\right)\right) \in\{0,1\}^{d}
$$

is componentwise decreasing, hence it follows from the Definition 3.4 and Lemma 3.3 a) that the process

$$
[0, \infty) \ni t \mapsto \hat{Y}^{e}(t):=\left(1_{\left[0, e_{1}\right)}\left(\Lambda_{1}(t)\right), \ldots, 1_{\left[0, e_{d}\right)}\left(\Lambda_{d}(t)\right)\right)
$$

is associated. In particular, for $k \in \mathbb{N}$ and times $0 \leq t_{1}<\ldots<t_{k}$ the $\mathbb{R}^{d k}$ valued vector $\left(\hat{Y}^{e}\left(t_{1}\right), \ldots, \hat{Y}^{e}\left(t_{k}\right)\right)$ is associated. For $e \leq e^{\prime}$ in $[0, \infty)^{d}$ we have $\left(\hat{Y}^{e}\left(t_{1}\right), \ldots, \hat{Y}^{e}\left(t_{k}\right)\right) \leq\left(\hat{Y}^{e^{\prime}}\left(t_{1}\right), \ldots, \hat{Y}^{e^{\prime}}\left(t_{k}\right)\right)$ componentwise. Hence, for every measurable, bounded and componentwise increasing $f: \mathbb{R}^{d k} \rightarrow \mathbb{R}$, the function

$$
[0, \infty)^{d} \ni e \mapsto \mathbb{E}\left[f\left(\hat{Y}^{e}\left(t_{1}\right), \ldots, \hat{Y}^{e}\left(t_{k}\right)\right)\right]
$$

is measurable and componentwise increasing. Since $\Lambda$ and the default thresholds $E=\left(E_{1}, \ldots, E_{d}\right)$ are independent, it follows that $\left(\hat{Y}\left(t_{1}\right), \ldots, \hat{Y}\left(t_{k}\right)\right)$ with

$$
\hat{Y}(t)=\left(\hat{Y}_{1}(t), \ldots, \hat{Y}_{d}(t)\right):=\left(1_{\left[0, E_{1}\right)}\left(\Lambda_{1}(t)\right), \ldots, 1_{\left[0, E_{d}\right)}\left(\Lambda_{d}(t)\right)\right), \quad t \geq 0,
$$

is a monotone mixture of $E$ according to Definition 3.2. By Lemma 3.3 $\mathrm{e}$ it follows that $\left(\hat{Y}\left(t_{1}\right), \ldots, \hat{Y}\left(t_{k}\right), E\right)$ is associated. Noting that $Y_{i}(t)=1-\hat{Y}_{i}(t)$ for all obligors $i \in\{1, \ldots, d\}$ and $t \geq 0$, it follows from Lemma 3.3 a that $\left(Y\left(t_{1}\right), \ldots, Y\left(t_{k}\right)\right)$ is associated. Hence, by Definition 3.4, the default indicator process $Y$ is associated.

(c) For every obligor $i \in\{1, \ldots, d\}$ and time horizon $n \in \mathbb{N}$ define the approximation

$$
\tau_{i, n}=\min \left\{\tau_{i}, n\right\}=\int_{0}^{n}\left(1-Y_{i}(s)\right) d s .
$$


It follows from Lemma 3.5 that $\left(\tau_{1, n}, \ldots, \tau_{d, n}\right)$ is associated. Since $\left(\tau_{1, n}, \ldots, \tau_{d, n}\right)$ converges to the vector of default times $\tau=\left(\tau_{1}, \ldots, \tau_{d}\right)$ as $n \rightarrow \infty$ and since every $\tau_{i}$ is finite by assumption, it follows from Lemma $3.3 \mathrm{~d}$ d) that $\tau$ is associated.

4.2. Association in Model 1. Next consider the special assumption made in Section 2 that the default hazard rate $\lambda_{i}(t)$ of obligor $i \in\{1, \ldots, d\}$ at time $t \geq 0$ is of the form $\lambda_{i}\left(t, \Psi_{t}, Y_{t}\right)$. We now elaborate on Lemma 4.1 by considering feedback effects, namely that defaults in the portfolio influence the default hazard rates of the remaining obligors.

Remark 4.2. Please note that in order to obtain general models we allow for mixing the sources of dependence. This may not be a good idea for practical models. Exploiting for example the mixture approach in Marshall and Olkin (1988) one can replace an Archimedean copula for the thresholds $E$ by using suitable default hazard rates and choose the random variables in $E$ independent.

Theorem 4.3. Assume the following:

(a) The environment process $\Psi$ is associated and has càdlàg paths.

(b) The default thresholds $E$ are associated and independent of $\Psi$.

(c) For every obligor $i \in\{1, \ldots, d\}$, the default hazard rate $\lambda_{i}:[0, \infty) \times \mathbb{R}^{m} \times$ $\{0,1\}^{d} \rightarrow[0, \infty)$ is jointly measurable and, for every $t \in[0, \infty)$, increasing in the other arguments.

(d) For every obligor $i \in\{1, \ldots, d\}$ and every default state $y \in\{0,1\}^{d}$, the default hazard rate $\lambda_{i}(\cdot, \cdot, y):[0, \infty) \times \mathbb{R}^{m} \rightarrow[0, \infty)$ is continuous.

Let $\Lambda$ denote the cumulative default hazard process

$$
\Lambda(t)=\left(\Lambda_{1}(t), \ldots, \Lambda_{d}(t)\right)=\left(\int_{0}^{t} \lambda_{i}(s, \Psi(s), Y(s)) d s\right)_{i=1, \ldots, d}, \quad t \geq 0,
$$

with the default indicator process $Y=\left(Y_{1}, \ldots, Y_{d}\right)$ given by (2.3). Then the process $(\Lambda, Y, \Psi)$ is well defined and associated. If, in addition, all obligors default eventually for large $t$, then the default times $\tau=\left(\tau_{1}, \ldots, \tau_{d}\right)$ given by $(2.2)$ are well defined and associated.

Remark 4.4. (a) Note that (4.1) defines $\Lambda$ in terms of $Y$, which by (2.3) is defined in terms of $\Lambda$. The proof clarifies this is not a circular definition.

(b) The proof below even shows that $(\Lambda, Y, \Psi,-E)$ is associated, meaning that for every $k \in \mathbb{N}$ and all times $0 \leq t_{1}<\ldots<t_{k}$ we have association of the $\mathbb{R}^{(2 d+m) k+d_{\text {-valued }} \text { random vector }}$

$$
\left(\Lambda\left(t_{1}\right), Y\left(t_{1}\right), \Psi\left(t_{1}\right), \ldots, \Lambda\left(t_{k}\right), Y\left(t_{k}\right), \Psi\left(t_{k}\right),-E\right) .
$$

Proof. For a fixed threshold vector $e=\left(e_{1}, \ldots, e_{d}\right) \in[0, \infty)^{d}$ define iteratively, for every $n \in \mathbb{N}$, the approximation $\Lambda_{n}^{e}$ of the cumulative default hazard process by

$$
\begin{aligned}
\Lambda_{n}^{e}(t) & =\left(\Lambda_{n, 1}^{e}(t), \ldots, \Lambda_{n, d}^{e}(t)\right) \\
& =\left(\int_{0}^{t} \lambda_{i}\left(s, \Psi(s), Y_{n-1}^{e}(s)\right) d s\right)_{i=1, \ldots, d}
\end{aligned}
$$

for $t \geq 0$, where the default indicator process is given by

$$
Y_{n-1}^{e}(t)=\left(1_{\left[e_{i}, \infty\right)}\left(\Lambda_{n-1, i}^{e}(t)\right)\right)_{i=1, \ldots, d}, \quad t \geq 0
$$


with $\Lambda_{0}^{e}(t):=0$ for $t \geq 0$. A jump of $Y_{n-1}^{e}$ at time $s$ can only influence the hazard rate $\lambda$ and therefore $\Lambda_{n}^{e}$ from time $s$ onwards. Hence, we see iteratively for every $k \in \mathbb{N}$, that all $\Lambda_{n}^{e}$ for $n \geq k$ agree up to the $k$-th jump of $Y_{k}^{e}$. Since there are at most $d$ jumps corresponding to the defaults of all $d$ obligors, $\Lambda^{e}:=\Lambda_{d}^{e}$ with $Y^{e}:=Y_{d}^{e}$ is the fixed point of the iteration, reached in at most $d$ steps. Since $(\Lambda, Y)=\left(\Lambda^{E}, Y^{E}\right)$, we see that the process is well defined.

We now want to prove that the process $\left(\Lambda^{e}, Y^{e}, \Psi\right)$ is associated. Since $\Lambda_{0}^{e}$ and therefore $Y_{0}^{e}$ are deterministic, $\left(\Lambda_{0}^{e}, Y_{0}^{e}, \Psi\right)$ is associated by assumption (a). To proceed inductively, assume for an $n \in\{1, \ldots, d\}$ that $\left(\Lambda_{n-1}^{e}, Y_{n-1}^{e}, \Psi\right)$ is associated. Then by assumption (c) and Lemma 3.3 a), it follows that the process

$$
[0, \infty) \ni s \mapsto\left(\lambda_{1}\left(s, \Psi(s), Y_{n-1}^{e}(s)\right), \ldots, \lambda_{d}\left(s, \Psi(s), Y_{n-1}^{e}(s)\right), \Psi(s)\right) \in[0, \infty)^{d} \times \mathbb{R}^{m}
$$

is associated. Lemma 3.5 then implies that the process $\left(\Lambda_{n}^{e}, \Psi\right)$ given via 4.2 is associated. Since the map

$$
[0, \infty)^{d} \ni\left(x_{1}, \ldots, x_{d}\right) \mapsto\left(1_{\left[e_{1}, \infty\right)}\left(x_{1}\right), \ldots, 1_{\left[e_{d}, \infty\right)}\left(x_{d}\right)\right) \in\{0,1\}^{d}
$$

is increasing, it follows from Lemma 3.3 a that $\left(\Lambda_{n}^{e}, Y_{n}^{e}, \Psi\right)$ is associated. Therefore, the limit $\left(\Lambda^{e}, Y^{e}, \Psi\right)$ is associated, and by Lemma 3.3 a the same is true for $\left(-\Lambda^{e},-Y^{e},-\Psi\right)$.

Fix $k \in \mathbb{N}$ and times $0 \leq t_{1}<\ldots<t_{k}$. Our next aim is to show that

$$
\left(-\Lambda\left(t_{1}\right),-Y\left(t_{1}\right),-\Psi\left(t_{1}\right), \ldots,-\Lambda\left(t_{k}\right),-Y\left(t_{k}\right),-\Psi\left(t_{k}\right)\right)
$$

is a monotone mixture of the default thresholds $E$ according to Definition 3.2. Consider $e \leq e^{\prime}$ in $[0, \infty)^{d}$. Then $Y_{0}^{e^{\prime}}(t) \leq Y_{0}^{e}(t)$ for all $t \in[0, \infty)$. To proceed inductively, assume for an $n \in\{1, \ldots, d\}$ that $\left(\Lambda_{n-1}^{e^{\prime}}(t), Y_{n-1}^{e^{\prime}}(t)\right) \leq\left(\Lambda_{n-1}^{e}(t), Y_{n-1}^{e}(t)\right)$ componentwise for all $t \in[0, \infty)$. By assumption (c) and (4.2), it follows that $\Lambda_{n}^{e^{\prime}}(t) \leq \Lambda_{n}^{e}(t)$ for all $t \in[0, \infty)$, hence $Y_{n}^{e^{\prime}}(t) \leq Y_{n}^{e}(t)$ for all $t \in[0, \infty)$ by (4.3). After $d$ steps we arrive at $\left(\Lambda^{e^{\prime}}(t), Y^{e^{\prime}}(t)\right) \leq\left(\Lambda^{e}(t), Y^{e}(t)\right)$ for all $t \in[0, \infty)$. Hence, for every measurable, bounded and componentwise increasing function $f$ : $\mathbb{R}^{(2 d+m) k} \rightarrow \mathbb{R}$, the function

$$
[0, \infty)^{d} \ni e \mapsto \mathbb{E}\left[f\left(-\Lambda^{e}\left(t_{1}\right),-Y^{e}\left(t_{1}\right),-\Psi\left(t_{1}\right), \ldots,-\Lambda^{e}\left(t_{k}\right),-Y^{e}\left(t_{k}\right),-\Psi\left(t_{k}\right)\right)\right]
$$

is componentwise increasing. To summarize, since the vector

$$
\left(-\Lambda^{e}\left(t_{1}\right),-Y^{e}\left(t_{1}\right),-\Psi\left(t_{1}\right), \ldots,-\Lambda^{e}\left(t_{k}\right),-Y^{e}\left(t_{k}\right),-\Psi\left(t_{k}\right)\right)
$$

is associated for every $e \in[0, \infty)^{d}$, since the default thresholds $E$ are associated and independent of $\Psi$ by assumption (b), since $(\Lambda, Y, \Psi)=\left(\Lambda^{E}, Y^{E}, \Psi\right)$, and since the vector in (4.4) is a monotone mixture of $E$, it follows from Lemma 3.3 e that the vector in (4.4) extended by $E$ is associated. Using Lemma 3.3 a) and Definition 3.4 it follows that the process $(\Lambda, Y, \Psi)$ together with $-E$ is associated. Lemma 4.1 (c) implies that the default times are associated.

4.3. Association in Model 2. Recall that in Model2 the cumulative default hazard processes are modeled directly and that defaults of one obligor may increase the cumulative default hazard process of the others. In what follows we say that a realvalued stochastic process $\left(X_{t}\right)$ on our probability space is stochastically continuous if for all $t \geq 0$ and $\varepsilon>0$

$$
\lim _{s \rightarrow t} \mathbb{P}\left(\left|X_{t}-X_{s}\right|>\varepsilon\right)=0
$$


Note that this notion allows for jumps of the process as long as these jumps are not at predefined deterministic time points. The paths of Lévy processes for example have this property. In the second model we obtain now the following result:

Theorem 4.5. Assume the following:

(a) The environment process $\Psi$ is associated and stochastically continuous and has $\mathbb{P}$-a.s. increasing paths converging to infinity as $t \rightarrow \infty$.

(b) The joint add-on process $\Gamma$ is associated and stochastically continuous and has $\mathbb{P}$-a.s. increasing paths.

(c) The default thresholds E are associated.

(d) The random quantities in (a), (b) and (c) are independent.

Then the cumulative default hazard process $\Lambda(t)=\left(\Lambda_{1}(t), \ldots, \Lambda_{d}(t)\right)$ is associated and thus $\tau=\left(\tau_{1}, \ldots, \tau_{d}\right)$ is associated.

Proof. Suppose a threshold vector $e=\left(e_{1}, \ldots, e_{d}\right) \in[0, \infty)^{d}$ is given and define for $n \in \mathbb{N}$ recursively for times $t \in[(l-1) / n, l / n)$ with $l \in \mathbb{N}$ the discrete approximation $\hat{\Lambda}_{n}(t)=\left(\hat{\Lambda}_{n, 1}(t), \ldots, \hat{\Lambda}_{n, d}(t)\right)$

$$
=\left(\Psi_{i}\left(\frac{\lfloor n t\rfloor}{n}\right)+\sum_{j \neq i} \sum_{\nu=1}^{\lfloor n t\rfloor-1} \Gamma_{i, j}\left(\frac{\lfloor n t\rfloor}{n}-\frac{\nu}{n}\right) 1_{\left\{\hat{\Lambda}_{n, j}\left(\frac{\nu}{n}\right) \geq e_{j}, \hat{\Lambda}_{n, j}\left(\frac{\nu-1}{n}\right)<e_{j}\right\}}\right)_{i=1, \ldots, d}
$$

In order to prove that $\hat{\Lambda}_{n}$ is associated, consider $k \in \mathbb{N}$ and $0=t_{0}<t_{1}<\cdots<t_{k}$. Again $\hat{\Lambda}_{n}$ is piecewise constant on the intervals $[(l-1) / n, l / n)$ with $l \in \mathbb{N}$ and we may assume w.l.og. that $t_{j}=j / n$ for all $j \in\{0, \ldots, k\}$. Next, we suppose that $\Gamma=\gamma$ is given and show by induction on $l \in\{0, \ldots, k\}$ that every vector

$$
\left(\Psi\left(t_{0}\right), \ldots, \Psi\left(t_{k}\right), \hat{\Lambda}_{n}\left(t_{0}\right), \ldots, \hat{\Lambda}_{n}\left(t_{l}\right)\right)
$$

is associated. For $l=0, \hat{\Lambda}_{n}\left(t_{0}\right)=\Psi(0)$, hence (4.6) is associated by assumption (a). Now suppose the statement holds true for $l \in\{0, \ldots, k-1\}$. The variable $\hat{\Lambda}_{n}\left(t_{l+1}\right)$ can be written as

$$
\hat{\Lambda}_{n}\left(t_{l+1}\right)=h_{l}\left(\Psi\left(t_{l+1}\right), \hat{\Lambda}_{n}\left(t_{0}\right), \ldots, \hat{\Lambda}_{n}\left(t_{l}\right)\right),
$$

where the function $h_{l}$ is given by

$$
\begin{aligned}
{[0, \infty)^{d} \times S_{l} \ni\left(\psi, x_{0}, \ldots, x_{l}\right) \mapsto } & \left.\mapsto \sum_{j \in\{1, \ldots, d\} \backslash\{i\}} \sum_{\nu=1}^{l} \gamma_{i, j}\left(\frac{l+1}{n}-\frac{\nu}{n}\right) 1_{\left[x_{j, \nu} \geq e_{j}, x_{j, \nu-1}<e_{j}\right]}\right)_{i=1, \ldots, d}
\end{aligned}
$$

with $\psi=\left(\psi_{1}, \ldots, \psi_{d}\right)$ and

$$
\begin{gathered}
S_{l}:=\left\{\left(x_{0}, \ldots, x_{l}\right) \mid x_{p}=\left(x_{1, p}, \ldots, x_{d, p}\right) \in[0, \infty)^{d}, x_{j, 0} \leq x_{j, 1} \leq \cdots \leq x_{j, l},\right. \\
j=1, \ldots, d\} .
\end{gathered}
$$

Since the functions $\gamma_{i, j}$ are increasing, the function $h_{l}$ is increasing (in the usual componentwise order) which proves that

$$
\left(\Psi\left(t_{0}\right), \ldots, \Psi\left(t_{k}\right), \hat{\Lambda}_{n}\left(t_{0}\right), \ldots, \hat{\Lambda}_{n}\left(t_{l+1}\right)\right)
$$


is associated according to Lemma 3.3 a), which implies the statement.

By assumption (b), the vector $\left(\Gamma\left(t_{0}\right), \ldots, \Gamma\left(t_{k}\right)\right)$ is associated. By assumption (d), it is independent of $\Psi$. In addition, the vector in $(4.6)$ is a monotone mixture of $\left(\Gamma\left(t_{0}\right), \ldots, \Gamma\left(t_{k}\right)\right)$. This implies by Lemma 3.3 (e), that

$$
\left(\Psi\left(t_{0}\right), \ldots, \Psi\left(t_{k}\right), \Gamma\left(t_{0}\right), \ldots, \Gamma\left(t_{k}\right), \hat{\Lambda}_{n}\left(t_{0}\right), \ldots, \hat{\Lambda}_{n}\left(t_{k}\right)\right)
$$

is associated. Recall that we still condition on $E$. But since $E$ is independent of $\Psi$ and $\Gamma$ and since

$$
\left(-\Psi\left(t_{0}\right), \ldots,-\Psi\left(t_{k}\right),-\Gamma\left(t_{0}\right), \ldots,-\Gamma\left(t_{k}\right),-\hat{\Lambda}_{n}\left(t_{0}\right), \ldots,-\hat{\Lambda}_{n}\left(t_{k}\right)\right)
$$

is a monotone mixture of $E$ and associated we obtain again with Lemma 3.3 (e), that the unconditioned vector in 4.10 is associated and thus by 3.3 a also the unconditioned vector in 4.6.

Next we show that

$$
\left(\hat{\Lambda}_{n}\left(t_{0}\right), \ldots, \hat{\Lambda}_{n}\left(t_{k}\right)\right) \rightarrow\left(\Lambda\left(t_{0}\right), \ldots, \Lambda\left(t_{k}\right)\right)
$$

in probability as $n \rightarrow \infty$. More precisely we show that for all $t \geq 0$ :

$$
\Lambda\left(\left(t-2 \frac{d}{n}\right)_{+}\right) \leq \hat{\Lambda}_{n}(t) \leq \Lambda(t)
$$

which then implies the convergence result, since the stochastic continuity of $\Psi$ and $\Gamma$ together with the fact that defaults are not at deterministic time points implies the stochastic continuity of $\Lambda$. Let us first look at the second inequality: It suffices to show this inequality for $t=\frac{l}{n}, l \in \mathbb{N}_{0}$ since $\Lambda$ is increasing. This can be done by induction. The statement is clear for $l=0$. From equation 4.5 it is clear that the induction step $l \mapsto l+1$ follows when we can show that

$$
\inf \left\{t \in\left[0, \frac{l}{n}\right) \mid \hat{\Lambda}_{n, j}(t) \geq e_{j}\right\} \geq \inf \left\{t \in\left[0, \frac{l}{n}\right) \mid \Lambda_{j}(t) \geq e_{j}\right\} .
$$

But this follows from the induction hypothesis. For the first inequality let $0:=\tau_{0} \leq \tau_{1} \leq \cdots \leq \tau_{d}$ be the ordered default time points of the obligors in the given model. Then it can be shown by induction that for $t \in\left[\tau_{l}, \tau_{l+1}\right)$ it holds that

$$
\Lambda\left(\left(t-2 \frac{l+1}{n}\right)_{+}\right) \leq \hat{\Lambda}_{n}(t)
$$

Altogether the statement then follows.

4.4. Implications of associated default times. The fact that the default times are associated enables us to compare a credit risk model to one where the cumulative default hazard processes are independent. For this purpose, we recall:

Definition 4.6. Let $X$ and $Y$ be real-valued random variables. Then $X$ is said to be smaller than $Y$ with respect to the usual stochastic order (notation $X \leq_{\mathrm{st}} Y$ ), if $\mathbb{P}(X>t) \leq \mathbb{P}(Y>t)$ for all $t \in \mathbb{R}$.

Suppose now that $\tau=\left(\tau_{1}, \ldots, \tau_{d}\right)$ is associated and denote by $\tau^{\perp}=\left(\tau_{1}^{\perp}, \ldots, \tau_{d}^{\perp}\right)$ the version with independent components but the same one-dimensional marginal distributions. Then Lemma 3.8 b] implies that

$$
\mathbb{E}\left[f\left(\tau^{\perp}\right)\right] \leq \mathbb{E}[f(\tau)]
$$


for all measurable, supermodular $f: \mathbb{R}^{d} \rightarrow \mathbb{R}$ for which the expectations exist. In particular this implies that for non-void $I \subset\{1, \ldots, d\}$ and arbitrary time points $\left\{t_{i}\right\}_{i \in I} \subset[0, \infty)$

$$
\mathbb{P}\left(\tau_{i}^{\perp}>t_{i} \text { for all } i \in I\right) \leq \mathbb{P}\left(\tau_{i}>t_{i} \text { for all } i \in I\right)
$$

and

$$
\mathbb{P}\left(\tau_{i}^{\perp} \leq t_{i} \text { for all } i \in I\right) \leq \mathbb{P}\left(\tau_{i} \leq t_{i} \text { for all } i \in I\right)
$$

because the corresponding indicator functions are supermodular. This means that the probabilities for joint early or late defaults do not decrease compared to the independent case. Moreover, it follows directly from Definition 4.6 and 4.14 respectively 4.15), that for every non-void $I \subset\{1, \ldots, d\}$

$$
\min _{i \in I} \tau_{i}^{\perp} \leq_{\mathrm{st}} \min _{i \in I} \tau_{i} \quad \text { and } \quad \max _{i \in I} \tau_{i} \leq_{\mathrm{st}} \max _{i \in I} \tau_{i}^{\perp},
$$

showing that associated default times have the tendency to happen closer together than independent ones (cf. Bäuerle (1997)).

For the default times $\tau_{1}, \ldots, \tau_{d}$ of the $d$ obligors let $\tau_{1: d} \leq \cdots \leq \tau_{d: d}$ denote the order statistics. Since

$$
\tau_{k: d}=\min _{\substack{I \subset\{1, \ldots, d\} \\|I|=k}} \max _{i \in I} \tau_{i} \quad \text { for } k \in\{1, \ldots, d\},
$$

every $\tau_{k: d}$ is an increasing function of $\left(\tau_{1}, \ldots, \tau_{d}\right)$. If $\left(\tau_{1}, \ldots, \tau_{d}\right)$ is associated, then Lemma 3.3 a implies that the order statistics $\left(\tau_{1: d}, \ldots, \tau_{d: d}\right)$ is associated, too.

Another way to justify that association is a notion of positive dependence is to look at dependence measures like linear correlation, Kendall's tau or Spearman's rho (for an axiomatic definition of dependence measures see Nelsen (2006), chapter $5)$. Take a pair $\left(\tau_{i}, \tau_{j}\right)$ of two arbitrary default times and denote by $\left(\tau_{i}^{\prime}, \tau_{j}^{\prime}\right)$ an independent copy, then Kendall's tau is defined by

$$
\rho_{\mathrm{K}}\left(\tau_{i}, \tau_{j}\right)=\mathbb{E}\left[\operatorname{sign}\left(\tau_{i}-\tau_{i}^{\prime}\right) \operatorname{sign}\left(\tau_{j}-\tau_{j}^{\prime}\right)\right]
$$

and Spearman's rho by

$$
\rho_{\mathrm{S}}\left(\tau_{i}, \tau_{j}\right)=\rho\left(F_{i}\left(\tau_{i}\right), F_{j}\left(\tau_{j}\right)\right)
$$

where $F_{i}$ and $F_{j}$ are the marginal distribution functions of $\tau_{i}$ and $\tau_{j}$ and $\rho$ is the usual linear correlation. In the case $\tau_{i}$ and $\tau_{j}$ are independent, all three dependence measures are zero, whereas they are non-negative when $\tau_{i}$ and $\tau_{j}$ are associated (this follows directly from the definition of association, see also Nelsen $(2006))$.

\subsection{Association of the default thresholds and the environment process.} Lemma 4.1 and Theorems 4.3 and 4.5 assume that the default thresholds $E=$ $\left(E_{1}, \ldots, E_{d}\right)$ and the environment process $\Psi$ are associated, respectively. Since $E$ is a random vector, this can be done using Definition 3.1. However, association is not a simple property to check. Therefore, it seems to be reasonable to give some examples. As seen in Section 3.2, association is a property of the copula and does not involve the marginal distributions. This remark seems to be important because in many models the thresholds are given in terms of a copula $C$ rather than a random vector $E$ (see McNeil et al. (2005), Chapter 9.6). A very common copula for example is the Gauss copula (see McNeil et al. (2005) p. $190 \mathrm{ff}$ ). It follows from Müller and Stoyan (2002) p. 146 and Theorem 3.10.18 that the Gauss copula with invertible covariance matrix $\Sigma$ is associated if and only if $\Sigma^{-1}$ has non-positive off-diagonal elements. 
In what follows we give a number of processes $\left(X_{t}\right)$ which are associated and of interest in the credit risk framework. For general Markov processes there is a characterization of association by Liggett (2005) via the generator of the process (see also Szekli (1995) p. 156). For association of Itô-diffusions see e.g. Herbst and Pitt (1991) or Bäuerle and Manger (2010). In what follows we suppose that $\left(X_{t}\right)$ is real-valued.

4.5.1. Processes with independent increments: If $\left(X_{t}\right)$ has independent increments, then $\left(X_{t}\right)$ is obviously associated. This includes Lévy processes and their deterministic time changes. In particular, every deterministic process is associated (For some recent results on dependence properties of Lévy processes see Bäuerle et al. (2008)).

4.5.2. Birth-and-death processes and relatives: If $\left(X_{t}\right)$ is a continuous-time Markov process with countable state space, then there exists a characterization of CIS in terms of the generator (see Szekli (1995), p. 98). Hence, in these cases $\left(X_{t}\right)$ is also associated. An important subclass where this is the case are birth-and-death processes. In view of Model 2 it is also interesting that the process

$$
\Gamma(t)=\gamma\left(t 1_{\{t \leq \sigma\}}+\sigma 1_{\{t>\sigma\}}\right), \quad t \geq 0,
$$

is associated, where $\gamma: \mathbb{R} \rightarrow \mathbb{R}_{+}$is an increasing function with $\gamma(0)=0$ and $\sigma$ is an exponentially distributed random variable. This follows since for $0<t_{1}<$ $\cdots<t_{k}$ we have that $\mathcal{L}\left(\Gamma\left(t_{k}\right) \mid \Gamma\left(t_{1}\right), \ldots, \Gamma\left(t_{k-1}\right)\right)$ is stochastically increasing in $\left(\Gamma\left(t_{1}\right), \ldots, \Gamma\left(t_{k-1}\right)\right)$.

4.5.3. Cox-Ingersoll-Ross (CIR) model: A popular interest rate model which is often used in credit risk frameworks for $\left(\Psi_{t}\right)$ is the CIR square-root diffusion which is defined as the unique strong solution of the stochastic differential equation

$$
d X_{t}=\alpha\left(\beta-X_{t}\right) d t+\sigma \sqrt{X_{t}} d W_{t}, \quad X_{0}=x_{0}>0,
$$

with parameters $\alpha, \beta, \sigma>0$. According to Lemma 3.8 (a), this process is associated, because it is even CIS as we will explain next. It follows from Karatzas and Shreve (1991) Proposition 5.2.18 that $x_{0} \leq x_{0}^{\prime}$ implies $X_{t} \leq_{\text {st }} X_{t}^{\prime}$ for all $t \geq 0$ where $\left(X_{t}\right)$ and $\left(X_{t}^{\prime}\right)$ are both solutions of the preceding CIR stochastic differential equation, the only difference being the initial condition $X_{0}=x_{0}$ and $X_{0}^{\prime}=x_{0}^{\prime}$.

4.5.4. GARCH processes: Another very important class of models for daily riskfactor return series are GARCH processes. GARCH processes are defined in discrete time and we thus extend them on $t \in \mathbb{R}$ by setting $X_{t}=X_{\lfloor t\rfloor}$. We restrict here to $\operatorname{GARCH}(1,1)$ processes which are defined as follows: Suppose $\left(Z_{t}\right)_{t \in \mathbb{Z}}$ is a sequence of i. i. d. random variables with existing expectation and variance and $\mathbb{E}\left[Z_{t}\right]=0$ and $\operatorname{Var}\left(Z_{t}\right)=1$. The $Z_{t}$ are called innovations. A prominent example are Gaussian innovations, i.e. $Z_{t} \sim \mathcal{N}(0,1)$. It holds that

$$
X_{t}=\sigma_{t} Z_{t}, \quad \sigma_{t}^{2}=\alpha_{0}+\left(\alpha_{1} Z_{t-1}^{2}+\beta\right) \sigma_{t-1}^{2}
$$

where $\alpha_{0}>0, \alpha_{1}, \beta \geq 0$. In order to obtain strict stationarity we have to assume that $\mathbb{E}\left[\log \left(\alpha_{1} Z_{t}^{2}+\beta\right)\right]<0$. The $\sigma_{t}^{2}$ are given explicitly by

$$
\sigma_{t}^{2}=\alpha_{0}+\alpha_{0} \sum_{i=1}^{\infty} \prod_{j=1}^{i}\left(\alpha_{1} Z_{t-j}^{2}+\beta\right) .
$$


Obviously $\sigma_{t}^{2}$ is increasing in $\left|Z_{s}\right|$ for all $s \in \mathbb{Z}$ and thus also $\left|X_{t}\right|=\sigma_{t}\left|Z_{t}\right|$. According to Lemma 3.3 a we obtain that the absolute value of a $\operatorname{GARCH}(1,1)$ process $\left(\left|X_{t_{1}}\right|, \ldots,\left|X_{t_{n}}\right|\right)$ is associated for all $t_{1}<\cdots<t_{n}$. In particular the volatility process is associated.

\subsection{Special models.}

4.6.1. Conditionally independent defaults. Suppose that in Model 1 the hazard rate $\lambda_{i}(t, \psi, y)$ depends only on $\psi$. These models are said to have conditionally independent defaults when the default time points are conditionally independent given $\mathcal{F}_{\infty}$, i.e.

$$
\mathbb{P}\left(\tau_{1} \leq t_{1}, \ldots, \tau_{d} \leq t_{d} \mid \mathcal{F}_{\infty}\right)=\prod_{i=1}^{d} \mathbb{P}\left(\tau_{i} \leq t_{i} \mid \mathcal{F}_{\infty}\right) .
$$

This is the case if and only if the default thresholds $E_{1}, \ldots, E_{d}$ are independent (cf. McNeil et al. (2005) Section 9.6.2). In most models of this type it is assumed that the default hazard rates are linear combinations of independent affine jumpdiffusions like for example

$$
\lambda_{i}\left(\Psi_{t}\right)=\lambda_{i, 0}+\sum_{j=1}^{p} \lambda_{i, j} \Psi_{j}^{\mathrm{syst}}(t)+\Psi_{i}^{\mathrm{id}}(t)
$$

where the factor weights $\lambda_{i, j}$ are non-negative and $\Psi_{j}^{\text {syst }}$ represent systematic risk factors, whereas $\Psi_{i}^{\mathrm{id}}$ is the individual risk factor for obligor $i$. In this case $\lambda_{i}\left(\Psi_{t}\right)$ is obviously increasing in $\Psi_{t}=\left(\Psi_{1}^{\text {syst }}(t), \ldots, \Psi_{p}^{\text {syst }}(t), \Psi_{1}^{\text {id }}(t), \ldots, \Psi_{d}^{\text {id }}(t)\right)$ as required by Theorem 4.3 . Thus, association of the environment process $\Psi$ implies association of the default times by Theorem 4.3 .

4.6.2. Model of Jarrow and Yu (2001) and extensions. One of the first models to incorporate interacting default hazard rates is the proposal by Jarrow and $\mathrm{Yu}$ (2001). We illustrate their model using the following special case: Suppose there are two obligors. A so-called primary one and a secondary one. The default hazard rate of the primary obligor depends on the environment process $\Psi$ only, whereas the default hazard rate of the second obligor depends on $\Psi$ and on the default state of the primary obligor. As an example the authors propose (all coefficients $a_{i j}$ are supposed to be non-negative)

$$
\begin{aligned}
& \lambda_{1}(t)=\lambda_{1}\left(\Psi_{t}, Y_{t}\right)=a_{10}+a_{11} \Psi_{t}, \\
& \lambda_{2}(t)=\lambda_{2}\left(\Psi_{t}, Y_{t}\right)=a_{20}+a_{21} \Psi_{t}+a_{22} 1_{\left\{Y_{1}(t)=1\right\}},
\end{aligned}
$$

where $\Psi$ could be the short rate of interest. This means that upon default of obligor 1 , the default hazard rate of obligor 2 increases. Theorem 4.3 then implies that the default times are associated whenever $\Psi$ and $E$ are associated. If $a_{22}=0$, then the correlation of default times comes from the common factor $\Psi$ only. Its influence depends on how much $\Psi$ varies itself. If $\Psi$ is deterministic, defaults are independent. In a symmetric relationship it is reasonable to assume that

$$
\begin{aligned}
& \lambda_{1}(t)=\lambda_{1}\left(\Psi_{t}, Y_{t}\right)=a_{10}+a_{11} \Psi_{t}+a_{12} 1_{\left\{Y_{2}(t)=1\right\}}, \\
& \lambda_{2}(t)=\lambda_{2}\left(\Psi_{t}, Y_{t}\right)=a_{20}+a_{21} \Psi_{t}+a_{22} 1_{\left\{Y_{1}(t)=1\right\}},
\end{aligned}
$$




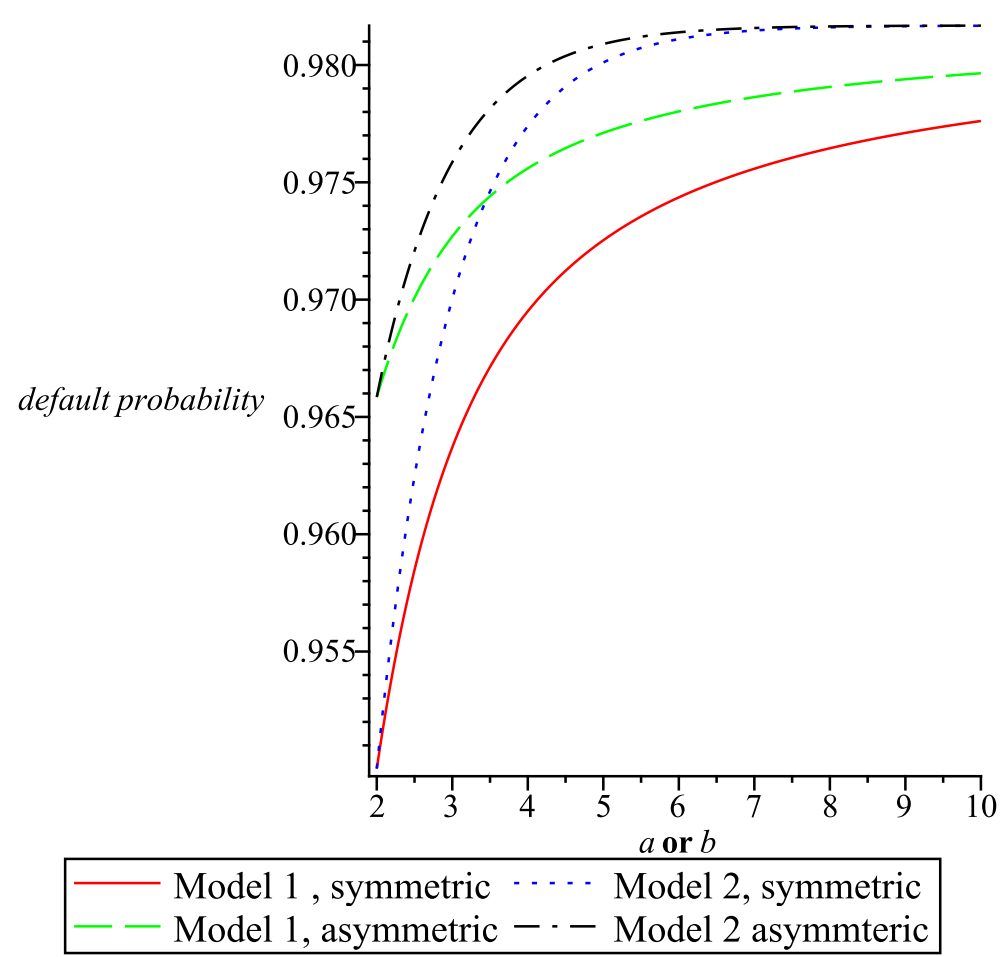

FiguRE 1. Default probability in both models, asymmetric case and symmetric case.

i.e. a default of obligor 2 also influences obligor 1 . Let us now modify the model in the following way where we use the cumulative hazard rate process:

$$
\begin{aligned}
& \Lambda_{1}(t)=a_{10}+a_{11} t+a_{12} \Psi_{t}+1_{\left\{\tau_{2} \leq t\right\}} \Gamma_{1,2}\left(t-\tau_{2}\right) \\
& \Lambda_{2}(t)=a_{20}+a_{21} t+a_{22} \Psi_{t}+1_{\left\{\tau_{1} \leq t\right\}} \Gamma_{2,1}\left(t-\tau_{1}\right) .
\end{aligned}
$$

The processes $\Psi$ and $\Gamma_{1,2}, \Gamma_{2,1}$ are stochastic processes with non-negative and increasing paths as in Model 2 . This time Theorem 4.5 implies that when $\Psi_{t}, \Gamma_{1,2}, \Gamma_{2,1}$ and $E$ are associated then the default times $\left(\tau_{1}, \tau_{2}\right)$ are associated.

In what follows we consider a simple numerical example which highlights that there is a difference between Model 1 and 2 as far as the strength of dependence is concerned which can be produced.

In our numerical example we have chosen $\lambda_{1}(t) \equiv 1, \lambda_{2}(t)=1+a 1_{\left\{Y_{1}(t)=1\right\}}$ in the asymmetric case and $\lambda_{1}(t)=1+a 1_{\left\{Y_{2}(t)=1\right\}}$ in the symmetric case. The parameter $a$ varies between 2 and 10 and the time horizon is two years. For Model 2 we have chosen $\Lambda_{1}(t)=t$ and $\Lambda_{2}(t)=t+1_{\left\{\tau_{1} \leq t\right\}} b$ in the asymmetric case and $\Lambda_{1}(t)=t+1_{\left\{\tau_{2} \leq t\right\}} b$ in the symmetric case. Here the parameter $b$ varies between 2 and 10 . 


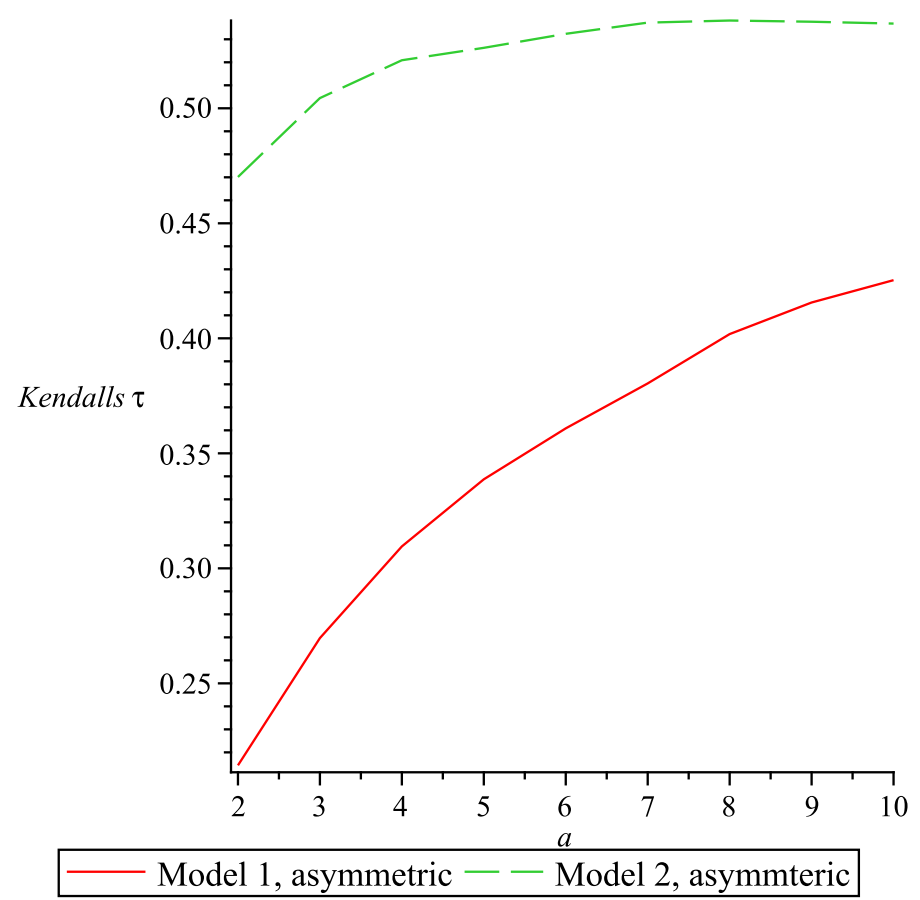

FiguRE 2. Kendall's tau in both models, asymmetric case.

Figure 1 shows the default probability (within the time horizon of two years) of obligor 2 in Model 1 (dashed line) and Model 2 (dotted-dashed line) in the asymmetric case as a function of $a$ and $b$ respectively and the default probability of both obligors in Model 1 (solid line) and Model 2 (dotted line) in the symmetric case (again as a function of $a$ and $b$ respectively). Obviously the default probability in Model 2 is in both cases larger than in Model 1. Next we choose the parameter $b$ such that in the asymmetric case the default probability of obligor 2 is the same in both Model 1 and 2. Since this default probability can be computed explicitly we arrive at

$$
b(a)=-\log \left(\frac{e^{-4}-e^{-2(1+a)}}{(a-1)\left(1-e^{-2}\right)}\right)-2, \quad a \in[2,10] .
$$

With this calibrated default probability (i.e. the default probability of obligor 1 and 2 are hence the same in Model 1 and 2) we derive by Monte Carlo simulation Kendall's tau between the default time points of the obligors conditioned on both default in the time interval $[0,2]$. The result is seen in Figure 2. The upper dashed line is Kendall's tau in Model 2, the lower solid line is Kendall's tau in Model 1. We observe that Model 2 produces a higher dependence in terms of Kendall's tau. In this model it is possible that both obligors default at the same time. However note that we cannot expect Kendall's tau to be too large even if $b$ is large because with probability $\frac{1}{2}$ we have $E_{1}>E_{2}$ under which condition there is no contagion. 


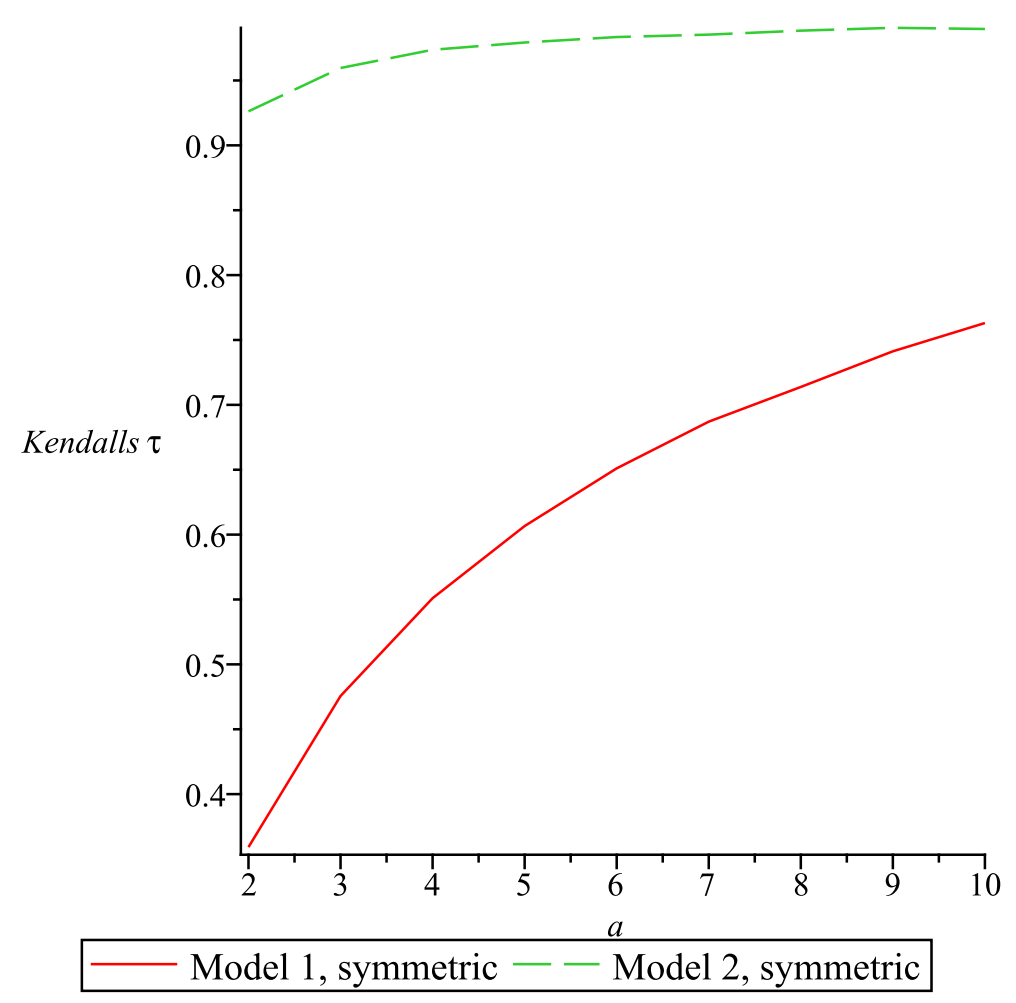

FiguRE 3. Kendall's tau in both models, symmetric case.

In Figure 3 we see Kendall's tau between the default time points of the obligors conditioned on both default in the time interval $[0,2]$ for the symmetric case. The parameter $b$ is again set as $b(a)$ as above to calibrate the default probabilities. The upper dashed line is Kendall's tau in Model 2, the lower solid line is Kendall's tau in Model 1. Again we observe that Model 2 produces a higher dependence in terms of Kendall's tau.

This simple example shows that Model 1 and 2 may produce quite different strength of dependence but also that features like the relation between obligors (is it symmetric or not) has a significant impact.

For a discussion of the sources of default correlation see e.g. Azizpour et al. (2010), Das et al. (2007) and Mai et al. (2011). Note that the investigation can be extended to the general hazard construction in $\mathrm{Yu}(2007)$ when appropriate assumptions on the hazard rate processes $\lambda_{i}\left(\cdot \mid I_{m}, T_{m}\right)$ are made. Moreover, the self-exciting model in Azizpour et al. (2010) which is based on a Hawkes process can be covered by Model 2 .

4.6.3. Model of Frey and Backhaus (2008). In Section 3 of their paper the authors propose a mean-field model with homogeneous groups. That is, they assume that the credit portfolio can be divided into $k$ groups and that the risks within a group 
are exchangeable. In particular the default hazard rate within a group is the same and depends on the proportion of defaulted obligors in this group so far. Formally if $K(r) \subset\{1, \ldots, d\}$ is the $r$-th group and $\bar{m}_{r}(y):=\frac{1}{|K(r)|} \sum_{j \in K(r)} 1_{\left\{y_{j}=1\right\}}$ the fraction of defaults, then the default hazard rate of a obligor $i \in K(r)$ is

$$
\lambda_{i}\left(t, \Psi_{t}, Y_{t}\right)=h_{r}\left(t, \Psi_{t}, \bar{m}_{1}\left(Y_{t}\right), \ldots, \bar{m}_{k}\left(Y_{t}\right)\right)
$$

where it is reasonable to assume that $h_{r}$ is increasing in $\bar{m}_{j}$ for all $j$. As a special example the authors consider an affine model with counterparty risk in which indeed $h_{r}$ is given by (all $a_{i j}$ are supposed to be $\geq 0$ )

$$
h_{r}(t, \psi, \bar{m})=\left[a_{r 0}+\sum_{j=1}^{m} a_{r j} \psi_{j}+a_{r \infty}\left(\sum_{j=1}^{k} \frac{|K(j)|}{d} \bar{m}_{j}-\sum_{j=1}^{k} \frac{|K(j)|}{d}\left(1-e^{-\bar{\lambda}_{j} t}\right)\right)\right]^{+},
$$

where $\bar{\lambda}_{j}$ is the expected default hazard rate of obligors in group $K(j)$. Obviously properties (c)-(d) of Theorem 4.3 are satisfied and thus, if $\left(\Psi_{t}\right)$ and $E$ are associated, then the default time points $\left(\tau_{1}, \ldots, \tau_{d}\right)$ are associated.

\section{Pricing of CRedit DeRivatives And the influence of DePendence}

5.1. Defaultable coupon bond without recovery. Suppose an obligor issues a coupon bond, which pays coupons $0<c_{1}, \ldots, c_{n}$ at time points $0<t_{1}, \ldots, t_{n}$ in case default has not happened so far. There is no recovery, i.e. if the obligor defaults, the coupon payment stops immediately. Let $\left(\Lambda_{t}\right)$ denote the cumulative default hazard process of the obligor and define the default time $\tau$ via (2.2). Let $R_{t} \geq 0$ denote the cumulative interest rate of a non-defaultable bond such that $e^{-R_{t}}$ is the stochastic factor for discounting from time $t$ to time 0 . Note that we apply the martingale modeling here, i.e. $\mathbb{P}$ is the equivalent martingale measure which is used for pricing. Moreover we assume that $E$ is independent from $\left(\Lambda_{t}\right)$ and $\left(R_{t}\right)$. Then the price $\pi_{c}^{0}$ of the corresponding defaultable coupon bond at time 0 is given by

$$
\pi_{c}^{0}=\sum_{k=1}^{n} c_{k} \mathbb{E}\left[e^{-R\left(t_{k}\right)} 1_{\left\{\tau>t_{k}\right\}}\right] .
$$

In case $\left(R\left(t_{k}\right), \Lambda\left(t_{k}\right)\right)$ are associated, the price can be bounded by the price of the corresponding non-defaultable coupon bond weighted with the probabilities of the coupon payment:

Lemma 5.1. If $\left(R\left(t_{k}\right), \Lambda\left(t_{k}\right)\right)$ are associated for $k=1, \ldots, n$, then

$$
\pi_{c}^{0} \geq \sum_{k=1}^{n} c_{k} \mathbb{E}\left[e^{-R\left(t_{k}\right)}\right] \mathbb{P}\left(\tau>t_{k}\right)
$$

Proof. Note that $\{\tau>t\}=\{\Lambda(t)<E\}$ and $\mathbb{P}(\Lambda(t)<E \mid \Lambda(t))=e^{-\Lambda(t)}$. By Lemma 3.3 a), the vector $\left(e^{-R\left(t_{k}\right)}, e^{-\Lambda\left(t_{k}\right)}\right)$ is associated for $k=1, \ldots, n$. Definition 3.1 directly implies

$$
\mathbb{E}\left[e^{-R\left(t_{k}\right)} 1_{\left\{\tau>t_{k}\right\}}\right]=\mathbb{E}\left[e^{-R\left(t_{k}\right)} e^{-\Lambda\left(t_{k}\right)}\right] \geq \mathbb{E}\left[e^{-R\left(t_{k}\right)}\right] \mathbb{E}\left[e^{-\Lambda\left(t_{k}\right)}\right],
$$

and the claim follows.

Remark 5.2. Assume the obligor has a default hazard rate process $\left(\lambda_{t}\right)$ and there is a positive spot rate $\left(r_{t}\right)$. Assume both processes have $\mathbb{P}$-a.s. càdlàg paths and $R_{t}=\int_{0}^{t} r_{s} d s$. If the $\mathbb{R}^{2}$-valued process $\left(\lambda_{t}, r_{t}\right)_{t \in[0, T]}$ is associated, then the vector 
$\left(R_{t}, \Lambda_{t}\right)$ is associated by Lemma 4.1 a and Lemma 5.1 applies. We just note here that up to now the literature does not give a clear picture whether interest rates and hazard default rates really influence each other in a positive way.

5.2. Collaterized debt obligations (CDOs). CDOs are a very important class of portfolio credit derivatives and we only consider a stylized form of it. To this end suppose we have a portfolio of $n$ obligors and

$$
L(T):=\sum_{i=1}^{n} L_{i} Y_{i}(T)
$$

is the total portfolio loss at time $T$ where the random variable $L_{i}$ is the loss given default of obligor $i$ and $Y_{i}(T)$ is as usual our default indicator. For simplicity we assume that the random variables $L_{1}, \ldots, L_{n}$ are independent and independent of all other random variables. By $K$ we denote the attachment point of the equity tranche, i.e. the notional of this tranche is immediately reduced in case a default occurs. Given any arbitrary default model, it follows from Lemma 3.8 b that when $\left(Y_{1}(T), \ldots, Y_{n}(T)\right)$ are associated, then the value of the premium leg of the equity tranche is bounded by

$$
\mathbb{E}(K-L(T))^{+} \geq \mathbb{E}\left(K-L^{\perp}(T)\right)^{+}
$$

where $L^{\perp}(T):=\sum_{i=1}^{n} L_{i} Y_{i}^{\perp}(T)$ and $\left(Y_{1}^{\perp}(T), \ldots, Y_{n}^{\perp}(T)\right)$ has same marginals as $\left(Y_{1}(T), \ldots, Y_{n}(T)\right)$ but the random variables are independent. For a senior tranche we get the reverse inequality. Of course this general bound may be quite worse. For a comprehensive analysis of CDO copula models including comparison results of CDO tranche premiums w.r.t. dependence parameters see Burtschell et al. (2008).

5.3. Credit swap contracts. In credit swap contracts three parties are involved: a reference party $D$ which issues a bond with a maturity $T^{*}$ and that is subject to default, a party $A$ which buys the bond, and a party $B$ which offers insurance against a default of $D$. More precisely, we assume that $A$ agrees to pay continuously a rate $\varrho$ to $B$ from time 0 up to the maturity $T \leq T^{*}$ of the swap and in exchange $B$ agrees to pay a certain amount of the loss to $A$ in case $D$ defaults. Besides $D$ also $A$ and $B$ may default. We make some idealizing assumptions: $A$ pays the swap rate $\varrho$ to $B$ until time $T$ or its own default, regardless of whether or not $B$ or $D$ have already defaulted. Also the compensation payment of $B$ to $A$ is at the maturity of the swap at time $T$ and is only paid if $B$ has not defaulted so far.

We assume a generic default model and denote by $\tau_{A}, \tau_{B}$ and $\tau_{D}$ the default times of the corresponding parties. Moreover, we denote by $\left(r_{t}\right)$ the spot rate process which is supposed to be $\left(\mathcal{G}_{t}\right)$-adapted and define $R_{t}=\int_{0}^{t} r_{s} d s$. The fair value at time $t \in[0, T]$ of $A$ 's swap rate payments with nominal value one is given by

$$
v_{A}(t):=\mathbb{E}\left[\int_{t}^{T} \exp \left(-\int_{t}^{s} r_{u} d u\right) 1_{\left\{\tau_{A}>s\right\}} d s \mid \mathcal{G}_{t}\right] .
$$

On the other hand, the fair value at time $t \in[0, T]$ of $B$ 's potential payment of $1 €$ at time $T$ in the event of $D$ 's default is

$$
v_{B}(t):=\mathbb{E}\left[\exp \left(-\int_{t}^{T} r_{u} d u\right) 1_{\left\{\tau_{D} \leq T\right\}} 1_{\left\{\tau_{B}>T\right\}} \mid \mathcal{G}_{t}\right] .
$$


The fair swap rate $\varrho$ for this contract starting at time 0 is thus given by

$$
\varrho=\frac{v_{B}(0)}{v_{A}(0)} .
$$

Obviously, when the swap rate is calculated at time 0 , the dependence between $A$ and $D$ and $A$ and $B$ is not relevant due to our assumptions. Only the dependence between $D$ and $B$ matters. We obtain the following result:

Lemma 5.3. Suppose $\varrho$ is the swap rate in case $\left(R_{T}, \tau_{D}, \tau_{B}\right)$ is associated and denote by $\varrho^{\perp}$ the swap rate when the data remains the same, except $\tau_{B}^{\perp}$ is now independent of $\left(R_{T}, \tau_{D}\right)$ and $\tau_{B} \stackrel{\mathrm{d}}{=} \tau_{B}^{\perp}$. Then $\varrho \leq \varrho^{\perp}$.

Proof. Note that we have

$$
1_{\left\{\tau_{D} \leq T\right\}} 1_{\left\{\tau_{B}>T\right\}}=1_{\left\{\tau_{D} \leq T\right\}}-1_{\left\{\tau_{D} \leq T\right\}} 1_{\left\{\tau_{B} \leq T\right\}}
$$

and

$$
\begin{aligned}
\mathbb{E}\left[e^{-R_{T}} 1_{\left\{\tau_{D} \leq T\right\}} 1_{\left\{\tau_{B} \leq T\right\}}\right] & =\mathbb{E}\left[e^{-R_{T}} e^{-\Lambda_{D}(T)} e^{-\Lambda_{B}(T)}\right] \\
& \geq \mathbb{E}\left[e^{-R_{T}} e^{-\Lambda_{D}(T)}\right] \mathbb{E}\left[e^{-\Lambda_{B}(T)}\right]
\end{aligned}
$$

where $\Lambda_{D}$ and $\Lambda_{B}$ are the cumulative default hazard processes of obligor $D$ and $B$ respectively. Following essentially the same arguments as in the proof of Lemma 5.1 yields the statement.

Remark 5.4. The preceding statement is in general wrong when we relax our model assumptions. Suppose for example that the compensation payment of $B$ to $A$ occurs immediately after the default of $D$ (if $B$ is alive). For simplicity assume that there is no interest, i.e. $r_{u} \equiv 0$. Then

$$
v_{B}(0)=\mathbb{E}\left[1_{\left\{\tau_{B}>\tau_{D}\right\}} 1_{\left\{\tau_{D} \leq T\right\}}\right] .
$$

In this case association of $\tau_{D}$ and $\tau_{B}$ does not imply a comparison with the independent case: Suppose for example $\tau_{D} \sim U(0, T)$ and $\tau_{B}=\tau_{D}$. Then obviously $\tau_{B}$ and $\tau_{D}$ are associated and $v_{B}(0)=\mathbb{P}\left(\tau_{B}>\tau_{D}\right)=0$ whereas in the independent case $v_{B}^{\perp}(0)=\mathbb{P}\left(\tau_{B}^{\perp}>\tau_{D}^{\perp}\right)=\frac{1}{2}$. On the other hand suppose $\tau_{D} \sim U(0, T-\varepsilon)$ for $\varepsilon>0$ small and $\tau_{B}=\tau_{D}+\varepsilon$. Again $\tau_{B}$ and $\tau_{D}$ are associated, but this time $v_{B}(0)=\mathbb{P}\left(\tau_{B}>\tau_{D}\right)=1$ whereas in the independent case $v_{B}^{\perp}(0)=\mathbb{P}\left(\tau_{B}^{\perp}<\tau_{D}^{\perp}\right)<1$.

5.4. $k$-th-to-default swaps. Now suppose that we have a portfolio of $d$ obligors and the bond defaults whenever the $k$-th default in the portfolio occurs. More precisely denote by $\tau_{1}, \ldots, \tau_{d}$ the default times of the $d$ obligors and by $\tau_{1: d} \leq \cdots \leq$ $\tau_{d: d}$ the order statistics. Then we have to replace $\tau_{D}$ in the preceding considerations by $\tau_{k: d}$. In this case the swap rate is given by

$$
\varrho_{k}=\frac{\mathbb{E}\left[\exp \left(-\int_{0}^{T} r_{u} d u\right) 1_{\left\{\tau_{k: d} \leq T\right\}} 1_{\left\{\tau_{B}>T\right\}}\right]}{\mathbb{E}\left[\int_{0}^{T} \exp \left(-\int_{0}^{s} r_{u} d u\right) 1_{\left\{\tau_{A}>s\right\}} d s\right]} .
$$

We obtain:

Lemma 5.5. Suppose $\varrho_{k}$ is the swap rate in case $\left(R_{T}, \tau_{1}, \ldots, \tau_{d}, \tau_{B}\right)$ is associated and denote by $\varrho_{k}^{\perp}$ the swap rate when the model is the same, except $\tau_{B}^{\perp}$ is independent of $\left(R_{T}, \tau_{1}, \ldots, \tau_{d}, \tau_{B}\right)$ with $\tau_{B} \stackrel{\mathrm{d}}{=} \tau_{B}^{\perp}$. Then $\varrho_{k} \leq \varrho_{k}^{\perp}$. 
Proof. The representation 4.17) shows that $\left(R_{T}, \tau_{k: d}, \tau_{B}\right)$ is an increasing function of $\left(R_{T}, \tau_{1}, \ldots, \tau_{d}, \tau_{B}\right)$ and therefore associated by Lemma 3.3 (a). The statement now follows from Lemma 5.3 .

\section{Conclusion}

The recent financial crisis has shown that dependencies between risks cannot be neglected. As far as credit risk is concerned in most (but not all) cases obligors show a positive dependence because they are opposed to the same macroeconomic factors, rely on the same resources or are related in their business. Thus reasonable credit risk models should be able to produce positive dependence between obligors. In this paper we have considered rather general reduced-form models and investigated under which conditions these models generate positive dependence in terms of association. Our study is only a qualitative one in the sense that we do not examine the degree of dependence. This has to be done for each specific model separately by investigating the influence of the model parameters. Here we can work with general models and can give hints on basic structural properties which should be satisfied. Moreover our results imply when simple models with independence assumptions on the random variables give upper or lower bounds on e.g. prices of credit derivatives. Some numerical examples highlight our findings.

\section{REFERENCES}

Azizpour, S., Giesecke, K., and Schwenkler, G. (2010) Exploring the sources of default clustering. preprint 1-27.

B ÄUERLE, N. (1997) Inequalities for stochastic models via supermodular orderings. Comm. Statis. Stochastic Models 13, 181-201.

B̈̈uerle, N., Blatter, A., and Müller, A. (2008) Dependence properties and comparison results for Lévy processes. Math. Methods Oper. Res. 67, 161-186.

BÄuerle, N. and Manger, A. (2010) Dependence properties of exit times with applications to risk management. Intern. J. Oper. Res. 7, 33-39.

Brigo, D. and CApponi, A. (2009) Bilateral counterparty risk valuation with stochastic dynamic models and application to credit default swaps. preprint 132 .

Burtschell, X., Gregory, J., and Laurent, J.-P. (2008) A comparative analysis of CDO pricing models. preprint 1-26.

Christofides, T. C. and Vaggelatou, E. (2004) A connection between supermodular ordering and positive/negative association. J. Multivariate Anal. 88, $138-151$.

Colangelo, A., Scarsini, M., and Shaked, M. (2005) Some notions of multivariate positive dependence. Insurance Math. Econom. 37, 13-26.

Das, S., Duffie, D., Kapadia, N., and Saita, L. (2007) Common failings: How corporate defaults are correlated. Journal of Finance 62, 93-118.

EBRAHimi, N. (2002) On the dependence structure of certain multi-dimensional Itô processes and corresponding hitting times. J. Multivariate Anal. 81, 128-137.

Esary, J. D., Proschan, F., and Walkup, D. W. (1967) Association of random variables, with applications. Ann. Math. Statist. 38, 1466-1474.

Frey, R. and BACKHAus, J. (2008) Pricing and hedging of portfolio credit derivatives with interacting default intensities. Int. J. Theor. Appl. Finance 11, 611634. 
Giesecke, K. and Weber, S. (2004) Cyclic correlations, credit contagion, and portfolio losses. J. Banking and Finance 28, 3009-3036.

Herbst, I. and PitT, L. (1991) Diffusion equation techniques in stochastic monotonicity and positive correlations. Probab. Th. Rel. Fields 87, 275-312.

Jakubowski, A. and Karlowska-Pik, J. (2011) Processes with block-associated increments. J. Appl. Probab. 48, 514-526.

JARrow, R. and YU, F. (2001) Counterparty risk and the pricing of defaultable securities. Journal of Finance 53, 2225-2243.

Joe, H. (1997) Multivariate Models and Dependence Concepts, volume 73 of Monographs on Statistics and Applied Probability. Chapman \& Hall, London.

Jogdeo, K. (1978) On a probability bound of Marshall and Olkin. Ann. Statist. 6, 232-234.

Karatzas, I. and Shreve, S. E. (1991) Brownian Motion and Stochastic Calculus, volume 113 of Graduate Texts in Mathematics. Springer-Verlag, New York, second edition.

KiJima, M. (1998) Monotonicities in a Markov chain model for valuing corporate bonds subject to credit risk. Math. Finance 8, 229-247.

Kou, S. and Peng, X. (2009) Default clustering and valuation of collaterized debt obligations. preprint $1-34$.

Liggett, T. M. (2005) Interacting Particle Systems. Classics in Mathematics. Springer-Verlag, Berlin. Reprint of the 1985 original.

MAI, J.-F. and Scherer, M. (2009) A tractable multivariate default model based on a stochastic time-change. Int. J. Theor. Appl. Finance 12, 227-249.

MAI, J.-F., Scherer, M., and ZAGst, R. (2011) CIID frailty models and implied copulas. preprint $1-35$.

Marshall, A. and Olkin, I. (1988) Families of multivariate distributions. Journal of the American Statistical Association 834-841.

McNeil, A. J., Frey, R., and Embrechts, P. (2005) Quantitative Risk Management. Princeton Series in Finance. Princeton University Press, Princeton, NJ. Concepts, techniques and tools.

Müller, A. and Stoyan, D. (2002) Comparison Methods for Stochastic Models and Risks. Wiley Series in Probability and Statistics. John Wiley \& Sons Ltd., Chichester.

Nelsen, R. B. (2006) An Introduction to Copulas. Springer Series in Statistics. Springer-Verlag, New York.

SzekLI, R. (1995) Stochastic Ordering and Dependence in Applied Probability, volume 97 of Lecture Notes in Statistics. Springer-Verlag, New York.

SzPIro, G. (2009) Eine falsch angewendete Formel und ihre Folgen - Unterschätzte Korrelation von Anlagewerten als Auslöser der Finanzkrise? Neue Zürcher Zeitung March, 18th.

YU, F. (2007) Correlated defaults in intensity-based models. Math. Finance 17, $155-173$. 
(N. Bäuerle) Institute for Stochastics, Karlsruhe Institute of Technology, D-76128 Karlsruhe, Germany

E-mail address: nicole.baeuerle@kit.edu

$U R L:$ http://www.math.kit.edu/stoch/ baeuerle/

(U. Schmock) Institute for Mathematical Methods in Economics, Vienna University of Technology, A-1040 Vienna, Austria

E-mail address: schmock@fam.tuwien.ac.at

$U R L:$ http://www.fam.tuwien.ac.at/ schmock/ 